\title{
Spinal Cord Injury: The Promise of Translational Research
}

\author{
Michael G. Fehlings, M.D., Ph.D., F.R.C.S.C., ${ }^{1-3}$ And Allyson Tighe, M.Sc. ${ }^{3}$ \\ ${ }^{1}$ Division of Genetics and Development, Toronto Western Research Institute; ${ }^{2}$ Division of Neurosurgery, \\ University of Toronto; ${ }^{3}$ Spinal Program, University Health Network, Toronto Western Hospital, Toronto, \\ Ontario, Canada
}

$\mathrm{S}$ pinal cord injury (SCI) is a condition with devastating consequences for the patient, family, and society. Although effective treatments for SCI remain limited, there have been many advances in recent years, which have promise for the future from a clinical translational perspective. This issue of Neurosurgical Focus explores some of the current basic science, preclinical, and clinical research directed towards this goal. Clinical investigations are also discussed with regard to the treatment and management of different types of SCI and of SCI in different populations. The issue concludes with a review of the current, ongoing, and planned clinical trials, providing a glimpse of the promising new therapies being developed for the treatment of SCI.

There are few clinical conditions as physically and psychologically devastating as traumatic SCI. The nature of the adult human central nervous system is such that recovery from a severe SCI is relatively uncommon, and most patients are left with a permanent disability. There are almost 300,000 people in North America living with SCI and over 20,000 new injuries every year. ${ }^{1,5}$ The treatment, care, rehabilitation, and community integration of these patients costs the health care system over $\$ 9.7$ billion a year in the US alone. ${ }^{1}$ The discovery and development of new treatments for SCI could substantially reduce the physical, psychological, and economic impact of this devastating condition.

The current treatments for SCI are limited. Patients generally undergo decompressive surgery and receive extensive rehabilitative therapy. Some may receive corticosteroids to reduce harmful inflammation at the site of injury, although this treatment remains controversial. ${ }^{3}$ None of these interventions are spectacularly effective in the recovery of function. Rehabilitation can lead to some improvements in motor control and sensation, but patients almost never recover the full use of the affected extremities or full autonomic function. In the past few years, however, there has been an explosion in the number of clinically relevant treatments being studied and developed for use in patients with SCIs. One of these therapeutic strategies, embodied by the Surgical Trial for Acute Spinal Cord Injury, examines the benefits of decompressive surgery within 24 hours of injury. The initial results from this study have been extremely positive and have the possibility of changing the surgical management of SCI. ${ }^{4}$ Another potential therapy, the Rho-antagonist Cethrin, has been shown to be effective in coaxing injured axons to grow in the hostile postinjury environment in rodent models of SCI; Phase I/IIa clinical trials in humans have just been completed with this drug. ${ }^{2}$ The results of subsequent clinical trials for this therapy will be watched with much anticipation. The international commitment to research of these and other therapies and treatments is offering hope for the SCI community around the world.

In this issue of Neurosurgical Focus, we delve into the exciting efforts being undertaken in the search for an effective treatment for SCI. We first review the groundbreaking regenerative research currently underway in animal models of SCI. The issue begins with a comprehensive review of the current state of basic animal studies and exciting new translational research. This review gives a fascinating glimpse into the future of SCI therapies. We then move on to look more specifically at several interesting topics in current animal studies, including the effects of glutamate blockage and corticosteroids on lipid peroxidation, as well as the transplantation of macrophages. Next, we explore clinical research and investigation. An overview of SCI in the geriatric population is provided, which has an important impact as the population ages and the demographic for SCI shifts towards the elderly. We also look at outcomes from traumatic medullary conus and cauda equina injuries, the cardiological complications of acute SCI, and the outcomes after different types of spinal cord trauma. We finish with a review of 
the current state of clinical and preclinical studies, providing perspective on where we are and how far we still have to go to achieve effective therapies for SCI.

Although we have come a long way in the management of chronic SCI, we are still unable to offer newly injured patients the promise of recovery. It is our hope that through the state of the art research that is currently being undertaken in laboratories, clinics, and operating rooms around the world, we will soon be able to provide hope for the future of these patients. The rate at which new discoveries are being made and translated into preclinical and clinical studies is thrilling. There is much excitement surrounding several interventions that are currently in clinical trials. The collaborative nature of these trials and other novel advances, including regenerative medicine, stem cell transplantation, cellular and molecular treatments, imaging, electrophysiology, and advances in surgical interventions and rehabilitation techniques, is allowing the brightest minds in SCI research to come to- gether and work towards a common goal: enabling SCI recovery.

\section{References}

1. Ackery A, Tator C, Krassioukov A: A global perspective on spinal cord injury epidemiology. J Neurotrauma 21:13551370, 2004

2. Baptiste DC, Fehlings MG: Update on the treatment of spinal cord injury. Prog Brain Res 161:217-233, 2007

3. Bracken MB: Steroids for acute spinal cord injury. Cochrane Database Syst Rev 3:CD001046, 2002

4. Fehlings MG, Maurais G, Theodore N, Harrop J, Kuntz C, Kwon B, et al: Results of a prospective, multicenter phase I/ IIa clinical trial to evaluate safety and preliminary efficacy of recombinant rho Inhibitor (Cethrin) in severe acute spinal cord injury. AANS Article ID:48829, 2008 (Abstract)

5. National Institute of Neurological Disorders and Stroke: Spinal Cord Injury: Hope Through Research. Bethesda, MD: NINDS, August 2003. NIH Publication No. 03-160 